\title{
On Fractional Frequency Reuse in Imperfect Cellular Grids
}

\author{
Patrick Mitran and Catherine Rosenberg \\ Department of Electrical and Computer Engineering \\ University of Waterloo
}

\begin{abstract}
Current point-to-multipoint systems suffer significant performance losses due to greater attenuation along the signal propagation path at higher frequencies, transmit power constraints of mobile users and base stations, and interference from neighboring cells. Fractional Frequency Reuse (FFR) is a technique to counteract these effects. Typically, the proposed FFR technique partitions a cell into a reuse 1 area, centered near the base-station and a reuse 3 area, located near the edges of the cell, with reuse 3 regions scheduled to minimize interference from neighboring cells. Unfortunately, virtually all analysis of FFR has been done under a perfect hexagonal lattice cellular grid, while no practical deployment has this degree of symmetry. In this paper we revisit the analysis of FFR for non-ideal cellular grids for cases with fading. We find that while for some non-ideal grids, a combination of reuse 1 and 3 is indeed optimal, for many others a combination of reuse 1 and 4 provide better performance. Thus, we conclude that for practical cellular layouts, the optimal re-use pattern for the edge of the cells is not necessarily 3 as commonly assumed, but is topology dependent.
\end{abstract}

Index Terms-Cellular Networks, Frequency Reuse, Fractional Frequency Reuse

\section{INTRODUCTION}

High data rate point-to-multipoint systems are now gaining widespread acceptance. Recent standards such as WiMAX (IEEE 802.16e) [1] and LTE [2] employ multiple access techniques based on OFDM. LTE for example, employs OFDMA on the downlink and Single-Carrier-FDMA, a form of precoded OFDMA with lower peak-to-average power ratio, on the uplink. Thus WiMAX and LTE allow users to be multiplexed by allocating time-frequency blocks to each user.

Among the challenges in providing high data rates and quality of service to mobile data users are: high path loss and greater signal attenuation due to higher frequencies, transmit power constraints at mobile users, and interference from neighboring cells.

Frequency reuse is a common technique to increase data throughput of point-to-multipoint systems. At one extreme, in a frequency reuse 1 system (FR(1)), each cell reuses the entire frequency band at the cost of creating possibly high interference. Among the advantages of such a scheme are that for users near the base-station, it can be shown that a very high rate per unit bandwidth is possible due to the large bandwidth available since those users do not typically suffer from significant interference. On the downside, for users near the edge of the cell, significant interference can result in low SINR, and thus only a low rate per unit bandwidth (and even potentially a lack of coverage) is achievable. Another alternative is a scheme such as frequency reuse $3(\mathrm{FR}(3))$. Here, each cell may use only one third of the system bandwidth, with neighboring cells using the remaining two thirds based on some coloring pattern. Cell-edge users now suffer significantly less interference and hence can achieve much higher rate per unit bandwidth, although users at the center of a cell do worse than in FR(1). Note that to use FR(3) a hexagonal lattice-like layout is necessary for cell placement (otherwise one cannot guarantee that a 3 color pattern can always be found). Under a set of simplistic assumptions (a perfect hexagonal grid for the placement of base stations, a $\log (1+$ SINR $)$ rate function and no fading), it can be shown that for each point in a cell, the rate per unit bandwidth, normalized to account for the reuse pattern, is either maximized by $\mathrm{FR}(1)$ or $\mathrm{FR}(3)$. We will show later that, this is not true under a set of more realistic assumptions, i.e., some users would do better with higher reuse factor $(\mathrm{e} . \mathrm{g}, 4,7,9, \ldots)$. It can also be shown that the total system throughput is maximized with FR(1) among all single frequency reuse schemes where the system throughput is defined as the sum of all user rates under a proportional fair scheduling policy. This is true even under a set of realistic assumptions, although this throughput maximization is done at the expense of edge users who receive low rates. Typically, cellular operators try to achieve a reasonable trade-off between throughput and coverage (i.e., the rate offered to edge users). In conventional cellular systems this trade-off is attained by selecting FR(3).

Even in next generation cellular systems, one of the main challenges is to find the right trade-off between the total system capacity (though there is no clear consensus on how this capacity should be defined) and coverage defined as the rate received by edge users ${ }^{1}$ while increasing the capacity using interference mitigation techniques. To obtain such a trade-off, one may consider a scheme using a mix of frequency reuse $r_{1}$ and $r_{2}>r_{1}$ [9], [3], commonly called fractional frequency reuse, or FFR. Roughly speaking, in an $\operatorname{FFR}\left(r_{1}, r_{2}\right)$ system, the frequency band $\mathcal{B}$ of the system (of size $B$ ) is divided into $r_{1}$ parts for $T_{1}$ per cent of the time and into $r_{2}$ parts for the rest of the time. Assuming time-sharing and a cycle of unit length, each cell is assigned one of the $r_{1}$ (resp. $r_{2}$ ) parts for communicating with core-cell (resp. edge-cell) users during $T_{1}$ (resp. $T_{2}$ ). It should be noted that an equivalent way to describe FFR is the following. We split the band $\mathcal{B}$ into 2 parts $\mathcal{B}_{1}$ and $\mathcal{B}_{2}$ of bandwidth $B_{1}$ and $B_{2}$ respectively. $\mathcal{B}_{1}$ (resp. $\mathcal{B}_{2}$ ) is further split into $r_{1}$ (resp. $r_{2}$ ) subbands. Each cell receives 2 subbands based on two distinct coloring patterns (one corresponding to $r_{1}$ and one to $r_{2}$ ), one subband of size $B_{1} / r_{1}$ and one of size $B_{2} / r_{2}$ with $B_{1}+B_{2}=B$. Here, all bands are used simultaneously without time-sharing.

Clearly one of the challenges is to choose $r_{1}$ and $r_{2}$, another one is to compute $B_{1}$ and another one is to decide which mode $r_{1}$ or $r_{2}$ to use to schedule users associated with a given cell. Two broad approaches to FFR are:

- static: users are scheduled by their base station in one of the 2 modes $r_{1}$ or $r_{2}$ based on their position, or path

\footnotetext{
${ }^{1}$ The term "edge user" should not be taken literally since with fading any user can potentially be an "unlucky" user irrespective of its position.
} 
loss,

- dynamic: in addition to position or path loss, cell load is taken into account to do the allocation of users to modes.

In view of the results showing that $\mathrm{FR}(1)$ and $\mathrm{FR}(3)$ are the best from an individual user's perspective (recall it was shown under a set of simplistic assumptions), many FFR studies in the literature select $r_{1}=1$ and $r_{2}=3$, i.e., $\operatorname{FFR}(1,3)$.

In this paper, we revisit this decision, i.e., we perform an in-depth study on FFR and show that under a set of realistic assumptions $\operatorname{FFR}(1,4)$ does significantly better than $\operatorname{FFR}(1,3)$ in many cases. This is particularly true when the cell deployment is not perfectly hexagonal, as in practice, since deployments are constrained by the topology of the environment, and are at best crudely approximated by hexagonal lattices. We model the base-station layout as a perturbed hexagonal grid. Two main messages of this study are 1) to emphasize the need for realistic and clearly posed assumptions and 2) to inform the 3GPP and LTE communities that FFR $(1,3)$ might not be the most robust and efficient FFR scheme.

The paper is organized as follows. Section II presents related work. Section III introduces the model, the problem formulation and some structural results. Section IV presents extensive numerical results and Section $\mathrm{V}$ concludes the paper.

\section{RELATED WORK}

The 3GPP-LTE has adopted a variant of FFR called Soft FFR (SFR) [9], [5], where the transmission power for the edgeusers and the center-users need not be the same, thus providing a degree of freedom that can be used to reduce co-channel interference.

In related work, [6] has studied the performance of 4 frequency allocation schemes, $\operatorname{FR}(1), \operatorname{FR}(3)$, static $\operatorname{FFR}(1,3)$, and dynamic FFR $(1,3)$, as well as proposed a new scheme called partial isolation. In [12] and [16], FFR comprising a mix of FR(1) and FR(3) was studied in the context of WiMAX, and was found to provide better throughput than FR(3) and better coverage than FR(1). In [15], the authors propose an Enhance FFR (EFFR) scheme that improves overall cell throughput over previous schemes such as SFR. In [10], an adaptive SFR (ASFR) scheme is proposed. The adaptive scheme allows the network configuration to track semi-static changes in user distribution and traffic, thus reducing call blocking probability. By comparison, [13] considers a selfish decentralized algorithm that permits the network to selforganize into efficient frequency reuse patterns. This is done in the context of constant bit rate traffic flows such as VoIP. In [8], the theoretical capacity and outage rate of OFDMA based FFR $(1,3)$ systems with Rayleigh fading is analyzed.

By far most work on analyzing cellular systems assumes a regular grid model such as hexagonal grids. Some work along the lines of modeling non-regular grids can be found in [4], [7] Specifically, [4] and [7] use tools from stochastic geometry to analyze network performance assuming base-station locations are placed according to a homogeneous Poisson point process (PPP). It is shown that the independence of the positions allows for some mathematical techniques to be employed. In [4], the probability of coverage and mean achievable rate for such a random network is derived under a simple FR scheme. These results are compared to results obtained from an actual base station deployment, and it is found that the PPP model is pessimistic, while the grid model is optimistic. In [7], these

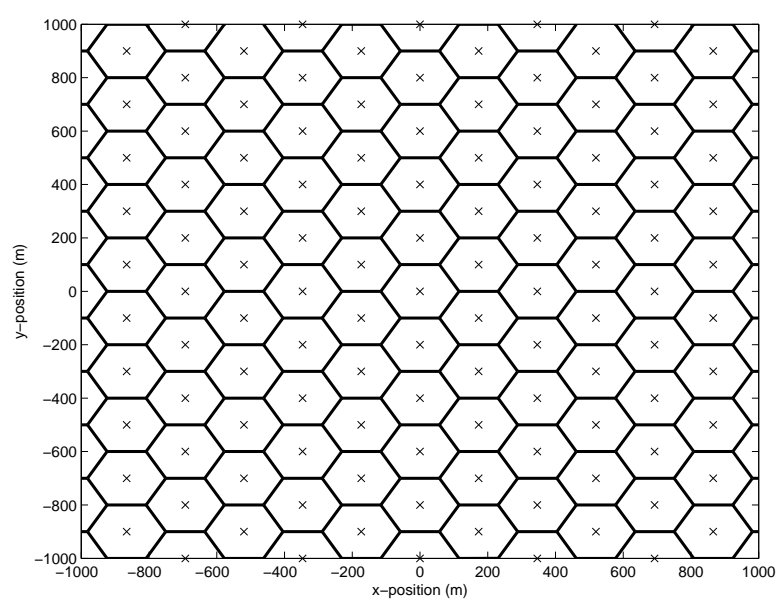

Fig. 1. Ideal hexagonal Voronoi regions for base station layout. Positions are in meters.

results are extended to $k$-tier heterogeneous networks, where each tier has its access points positioned according to an independent homogeneous PPP with possibly different density.

\section{Model And Problem Formulation}

In this section, we will present the system under consideration and the assumptions under which the study is performed.

\section{A. Base Station Placement}

We consider a cellular system comprised of a large number of base stations operating in downlink. In an ideal network, the base stations would be located at the center of a hexagonal lattice, as illustrated in Fig. 1.

In practice, base stations cannot always be located at the ideal positions to create a hexagonal lattice. If the ideal position of the $k$ th base station is $\mathbf{u}_{k}$ on the hexagonal grid, we model the actual position of the $k$ th base station to be

$$
\mathbf{X}_{k}=\mathbf{u}_{k}+\mathbf{Z}_{k},
$$

where $\mathbf{Z}_{k}$ is a random perturbation that is independent and identically distributed among the base stations. In particular, we take $\mathbf{Z}_{k}$ to be a uniform random variable on a disk of radius $0 \leq \mu \times R$ where $0 \leq \mu<1$ is a parameter that represents the degree to which the placement is non ideal, and $R$ is the inner radius of an ideal hexagonal cell (the case $\mu=0$ corresponds to the ideal case). Fig. 2 illustrates a typical layout and the corresponding Voronoi regions when $\mu=0.9$ is chosen.

For an ideal hexagonal lattice, a frequency reuse factor $r$ is feasible if $r$ can be written as $r=i^{2}+i j+j^{2}$, for $i, j \in \mathbb{N}$. Specifically, $i=1, j=0$ yields reuse $r=1 ; i=1, j=$ 1 yields reuse $r=3$, etc. The first few reuse factors for a hexagonal lattice are $1,3,4,7,9,12$, etc.

It should be noted that by placing the base-stations at perturbed positions compared to an ideal hexagonal grid, the same frequency reuse patterns are feasible. By contrast, for a base station placement according to a Poisson point process (PPP), the resulting Voronoi regions may not allow for reuse pattern 3 with no two neighboring cells sharing the same band as 4 is the chromatic number of the plane. Hence in a system with random placement, $\operatorname{FFR}(1,3)$ might not be feasible. 


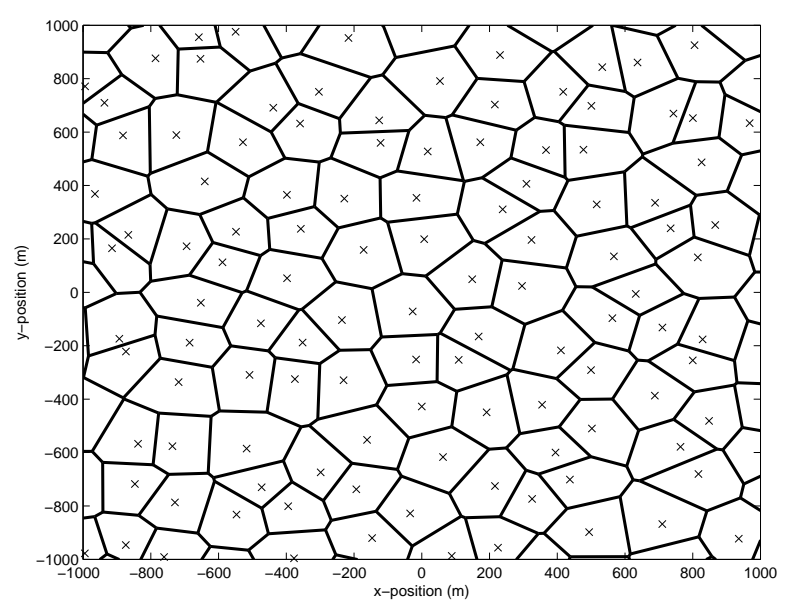

Fig. 2. Typical non-ideal Voronoi regions for perturbed base station layout with $\mu=0.9$.

\section{B. Channel and Interference Models}

We assume that the system operates in a given frequency band $\mathcal{B}$ of bandwidth $B$. Given a user $\ell$ in the region covered by the system, we assume that it will associate to the nearest base station. If at a given instant, the set of base stations (other than the one to which $\ell$ is associated) that are transmitting in the same frequency subband that user $\ell$ is receiving in is denoted by $\mathcal{I}_{\ell}$, then user $\ell$ will observe a signal to interference and noise ratio (SINR) $\gamma_{\ell}$ given by

$$
\gamma_{\ell}=\frac{P_{k(\ell)} \times g_{k(\ell), \ell}}{N_{0}+\sum_{k \in \mathcal{I}_{\ell}} P_{k} \times g_{k, \ell}},
$$

where user $\ell$ is associated with base station $k(\ell)$, base station $k$ transmits at power $P_{k}, g_{k, \ell}$ is the power gain between base station $k$ and user $\ell$, and $N_{0}$ denotes the receiver noise. Specifically, $g_{k, \ell}$ is in general a combination of path loss and fading,

$$
g_{k, \ell}=G_{k, \ell} \times P L\left(d_{k, \ell}\right),
$$

where $G_{k, \ell}$ is the power gain due to fading, $d_{k, \ell}$ is the distance between base station $k$ and user $\ell$, and

$$
P L(d)=\left\{\begin{array}{cc}
\left(\frac{\lambda}{4 \pi d}\right)^{2} & d<d_{0} \\
\left(\frac{\lambda}{4 \pi d_{0}}\right)^{2}\left(\frac{d}{d_{0}}\right)^{\eta} & d \geq d_{0}
\end{array},\right.
$$

where $\lambda$ is the wavelength, $d_{0}$ is the near field distance, and $\eta$ is the path loss exponent [11].

We assume that the rate function is logarithmic. More precisely, given that user $\ell$ has $\operatorname{SINR} \gamma_{\ell}$, and that cell $k$ is assigned bandwidth $b$, the instantaneous rate at which base station $k$ may communicate with user $\ell$ is $b \log _{2}\left(1+\gamma_{\ell} / \Gamma\right)$, where $\Gamma$ models the coding gap to Shannon capacity [14].

\section{FFR Scheme}

As the base station locations are perturbed from an ideal hexagonal lattice, frequency reuse patterns of $r=$ $1,3,4,7,9, \ldots$, can still be constructed as discussed earlier.

Let us first consider the scheme $\operatorname{FR}(r)$, i.e., a scheme using a simple reuse factor $r$. Let $I_{k(\ell), \ell, r}$ denote the aggregate interference from all other base stations in the network to user

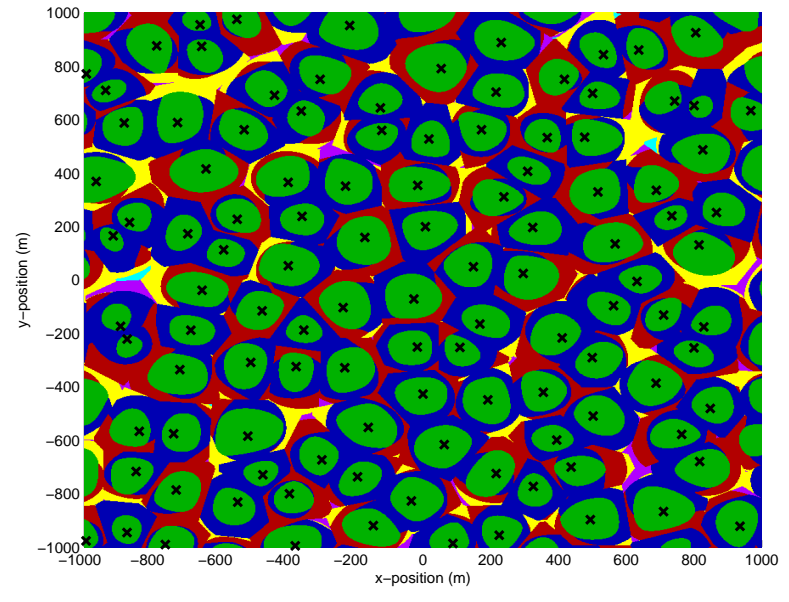

Fig. 3. Optimal frequency reuse factor as a function of user position for cell layout of Fig. 2. Base station positions are denoted by $\times$. Color assignment: Green:1, Blue:3, Red:4, Yellow:7, Purple:9, Cyan:12

$\ell$ who is associated with base station $k(\ell)$ assuming frequency reuse factor $r$. Then, given that cell $k$ will be allocated a bandwidth $B / r$, the instantaneous rate seen by $\ell$ is

$$
\rho_{\ell, r}=\frac{B}{r} \log _{2}\left(1+\frac{P_{k(\ell)} \times g_{k, \ell}}{\Gamma \times\left(N_{0}+I_{k(\ell), \ell, r}\right)}\right),
$$

and the instantaneous rate per unit bandwidth, normalized to account for the re-use pattern, is thus $\rho_{\ell, r} / B$.

As discussed earlier, there is a tradeoff for using a frequency reuse factor $r$ [9]. Namely, while increasing the reuse factor results in less interference which may increase the instantaneous rate per $\mathrm{Hz}$, it requires partitioning the bandwidth into finer subbands, which may decrease the effective rate per $\mathrm{Hz}$.

It is well known that for a regular hexagonal lattice without fading, frequency reuse 1 is optimal (in that it offers the highest rate per unit bandwidth) for users near the base station, while frequency reuse 3 is optimal for users near the edge of the cell. For the case of irregular deployments such as the one in Fig. 2 things are less clear. Fig. 3 illustrates the frequency reuse factor that optimizes the instantaneous rate per unit bandwidth, $\rho_{\ell, r} / B$, as a function of the user position for the cell layout of Fig. 2, assuming a path loss only model (i.e., no fading). Given the irregular structure of the layout, frequency reuse factors of up to 12 can be optimal for some locations. From the figure, it is apparent that there are significant regions where frequency reuse factors greater than 3 are desirable. Furthermore, it has been observed that for regions where frequency reuse of 3 is optimal, a frequency reuse of 4 did not result in a significantly smaller rate.

We now consider an $\operatorname{FFR}\left(r_{1}, r_{2}\right)$ scheme, i.e., we split the band $\mathcal{B}$ into 2 parts $\mathcal{B}_{1}$ and $\mathcal{B}_{2}$ of bandwidth $B_{1}$ and $B_{2}$ respectively. $\mathcal{B}_{1}$ (resp. $\mathcal{B}_{2}$ ) is further split into $r_{1}$ (resp. $r_{2}$ ) subbands. Each cell receives 2 subbands based on two distinct coloring patterns (one corresponding to $r_{1}$ and one to $r_{2}$ ), one subband of size $B_{1} / r_{1}$ and one of size $B_{2} / r_{2}$ with $B_{1}+$ $B_{2}=B$. Here, all bands are used simultaneously without time-sharing. Clearly one of the challenges is to choose $r_{1}$ and $r_{2}$, another one is to compute $B_{1}$ and another one is to decide which mode $r_{1}$ or $r_{2}$ to use to schedule users associated with a given cell. 


\section{Problem Formulation}

It is important to note that the rate seen by a user $\ell$ associated to base station $k$ will not only depend on the mode selected by the base station to schedule $\ell$ but also on the scheduling policy being used. Many scheduling policies have been proposed but the most common and realistic one is based on proportional fairness (PF). In a single cell that has been allocated a certain bandwidth, PF scheduling effectively results in allocating the same time to each user independent of its position, i.e., each user will receive a different rate based on its channel condition.

More precisely, if the rate assigned to user $\ell$ is $\Lambda_{\ell}$ then the corresponding PF utility is $\tilde{U}=\sum_{\ell=1}^{L} \ln \Lambda_{\ell}$, where $L$ denotes the number of users in the cell. Equivalently, optimizing the utility $\tilde{U}$ results in the same allocation as optimizing the geometric mean $U$ of the rates. We will use $U$ as the objective function in the following.

$$
U=\left(\prod_{\ell=1}^{L} \Lambda_{\ell}\right)^{1 / L}
$$

In the following, we compare $\operatorname{FFR}(1,3)$ with $\operatorname{FFR}(1,4)$ when, based on the utility in (6), we allocate optimally each user to one of the two modes and we compute $B_{1}$ optimally. Recall that each user is associated to the closest base station. We base our computation of $B_{1}$ on the worst case cell, i.e., we consider the largest cell in the middle of a region in the center of the network.

Let $\mathcal{C}$ be this cell and $L$ be the number of users in $\mathcal{C}$ which without loss of generality are assumed to be indexed as $\ell=$ $1, \ldots, L$. We assume that $\mathcal{C}$ has been allocated a band $\beta_{1}$ of size $B_{1} / r_{1}$ for mode $r_{1}$ and a subband $\beta_{2}$ of size $B_{2} / r_{2}$ for mode $r_{2}$. Let $x_{\ell}^{1}=1$ if user $\ell$ is scheduled in subband $\beta_{1}$ and $x_{\ell}^{1}=0$ if user $\ell$ is scheduled in subband $\beta_{2}$. Then the problem can be formulated as follows for $\operatorname{FFR}\left(r_{1}, r_{2}\right)$ :

$$
\begin{aligned}
& \max _{B_{1},\left\{x_{\ell}^{1}\right\}}\left(\prod_{\ell=1}^{L} \Lambda_{\ell}\right)^{1 / L} \\
& \Lambda_{\ell}=x_{\ell}^{1} B_{1} R_{\ell, r_{1}}+\left(1-x_{\ell}^{1}\right)\left(B-B_{1}\right) R_{\ell, r_{2}} \\
& x_{\ell}^{1} \in\{0,1\} \quad \forall \ell \in \mathcal{C} \\
& 0 \leq B_{1} \leq B,
\end{aligned}
$$

where $R_{\ell, r_{j}}(j=1,2)$ is defined below:

$$
R_{\ell, r_{j}}=\frac{1}{r_{j}} \log _{2}\left(1+\frac{P \times g_{\ell}}{\Gamma \times\left(N_{0}+I_{\ell, r_{j}}\right)}\right) .
$$

Here, $P$ is the transmit power of the base station in $\mathcal{C}, g_{\ell}$ is the power gain between this base station and $\ell \in \mathcal{C}$, and $I_{\ell, r_{j}}$ is the aggregate interference from all other base stations in the system that use $\beta_{j}$.

The following Proposition provides some structure to the above problem.

Proposition 1: Given $B_{1}$, the optimal proportional fair allocation of users in $\mathcal{C}$ to modes has the following structure. There is a real number $t$, such that for all users $\ell$ in cell $\mathcal{C}$, if $R_{\ell, r_{1}} / R_{\ell, r_{2}}>t$, then user $\ell$ is assigned to mode $r_{1}$, and is assigned to mode $r_{2}$ otherwise. Furthermore, if $L_{1}$ and $L_{2}$ denote the number of users assigned to mode $r_{1}$ and $r_{2}$ respectively, then users assigned to reuse pattern $r_{j}$ are each allocated bandwidth $\left(B_{j} / r_{j}\right) / L_{j}$.
Proof: Consider the utility when users $\ell_{1}$ and $\ell_{2}$ are assigned to reuse patterns $r_{1}$ and $r_{2}$ respectively, and $b_{\ell}$ is the bandwidth assigned to user $\ell$, and denote this as case 'a':

$$
U_{a}=\left(\prod_{\ell} b_{\ell} \times \prod_{\ell \neq \ell_{1}, \ell_{2}} R_{\ell, r(\ell)} \times R_{\ell_{1}, r_{1}} \times R_{\ell_{2}, r_{2}}\right)^{(1 / L)},
$$

where $r(\ell)$ is the mode allocated to user $\ell$, i.e., $r(\ell)=x_{\ell}^{1} r_{1}+$ $\left(1-x_{\ell}^{1}\right) r_{2}$. Now, consider the utility obtained by swapping the reuse pattern (and bandwidth allocation) of users $\ell_{1}$ and $\ell_{2}$, and denote this as case ' $b$ '. Then the utility is

$$
U_{b}=\left(\prod_{\ell} b_{\ell} \times \prod_{\ell \neq \ell_{1}, \ell_{2}} R_{\ell, r(\ell)} \times R_{\ell_{1}, r_{2}} \times R_{\ell_{2}, r_{1}}\right)^{(1 / L)} .
$$

Hence, case 'a' provides better utility than case 'b' provided

$$
R_{\ell_{1}, r_{1}} / R_{\ell_{1}, r_{2}}>R_{\ell_{2}, r_{1}} / R_{\ell_{2}, r_{2}} .
$$

Specifically, denoting by $\alpha_{\ell}:=R_{\ell, r_{1}} / R_{\ell, r_{2}}$, we find that for an optimal resource allocation scheme, if $\ell_{1}$ and $\ell_{2}$ are users in reuse pattern $r_{1}$ and $r_{2}$ respectively, then $\alpha_{1}>\alpha_{2}$. Hence, there must exist a threshold $t$ such that $\alpha_{\ell}>t$ iff user $\ell$ is assigned reuse pattern $r_{1}$.

Finally, it follows that a user assigned to reuse pattern $r_{j}$ is allocated $\left(B_{j} / r_{j}\right) / L_{j}$ bandwidth since the product $\prod_{i=1}^{K} z_{i}$ subject to the constraints $\sum z_{i} \leq\left(B_{j} / r_{j}\right), z_{i} \geq 0$ is maximized when $z_{i}=\left(B_{j} / r_{j}\right) / K$.

The significance of the above structural result is that given $B_{1}$ and the appropriate threshold $t$, the optimal allocation of users to modes is simple to compute. Thus, for a given $B_{1}$, it remains only to compute the threshold $t$, which can be found by numerical search. This search is simple as the threshold $t$ is only used to partition the $\alpha$ 's into those greater that $t$ and those not. Thus, we can restrict the search for $t$ to $t=\alpha_{1}, \alpha_{2}$, .... We can then search for the best bandwidth allocation by varying $B_{1}$ from 0 to $B$ in small increments.

\section{RESULTS}

We first describe the ideal, non-perturbed network topology. Here, we consider a hexagonal lattice network with a base station placed at the origin, and an additional 15 rings of base stations around this first base station for a total of 721 cells. The inner radius of a hexagonal cell is $100 \mathrm{~m}$, and thus the distance between two base stations is $D=200 \mathrm{~m}$. Hence, the total region is approximately a hexagon of inner radius $3.1 \mathrm{~km}$.

The system bandwidth $B=20 \mathrm{MHz}$, the path loss exponent is selected as $\eta=3.7$, the coding gap is taken to be $\Gamma=3$ $\mathrm{dB}$, the near field distance is $d_{0}=10 \mathrm{~m}$, and $\lambda=0.3 \mathrm{~m}$, which corresponds to a carrier frequency of approximately $1 \mathrm{GHz}$. We assume the network is interference limited, and thus take $N_{0}=0$ for simplicity. All base stations employ the same normalized transmission power $P=P_{k}=1$. Rayleigh fading, when considered, results in a gain $G_{k, \ell}$ that is independent and identically distributed between pairs $(k, \ell)$ with an exponential distribution with mean 1, i.e., $P\left[G_{k, \ell} \leq x\right]=1-e^{-x}$.

Base stations are then randomly perturbed as described in Section III-A, either with $\mu=0$ (no perturbation), or with $\mu=$ 0.9 . We focus on an inner region at the center corresponding to a rectangular region from $x=y=-1000 \mathrm{~m}$ to $x=y=$ 


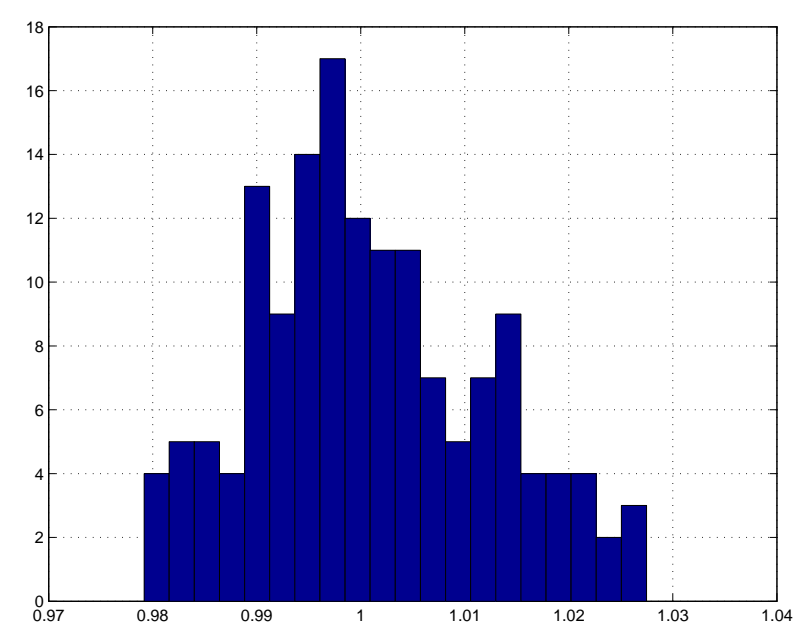

Fig. 4. Histogram of gain in geometric mean of user rates for $\operatorname{FFR}(1,4)$ over $\operatorname{FFR}(1,3)$ for no pertubation $(\mu=0)$ with 150 different fading realizations.

$1000 \mathrm{~m}$. We assume that users are placed on a rectangular grid with a spacing of $10 \mathrm{~m}$.

We are interested in studying the performance of the users when the system employs $\operatorname{FFR}(1,3)$, compared to when it uses FFR(1,4), under proportional fair scheduling, i.e., we will solve problem (7) for both schemes and compare the value of their objective function, i.e., the geometric mean obtained when optimizing both $B_{1}$ and the user allocation to modes.

We first consider no perturbation of the base station placement and a path loss only model, (i.e., $G_{k, \ell}=1$, no fading). Here, as in [9], $\operatorname{FFR}(1,3)$ provides the best performance. A geometric mean of $103.1 \mathrm{kbit} / \mathrm{s}$ per user for $\operatorname{FFR}(1,3)$ compared to $100.6 \mathrm{kbit} / \mathrm{s}$ per user for $\operatorname{FFR}(1,4)$ is found.

We next consider no perturbation of the base station placement but with Rayleigh fading, for 150 different fading realizations. Interestingly, we find that for a perfect hexagonal lattice, $\operatorname{FFR}(1,3)$ outperforms $\operatorname{FFR}(1,4)$ in terms of the geometric mean of the user rates only $51.3 \%$ of cases. Fig. 4 shows a histogram of the gain in geometric mean of the rates for $\operatorname{FFR}(1,4)$ over $\operatorname{FFR}(1,3)$ for the 150 realizations, confirming that neither $\operatorname{FFR}(1,3)$ nor $\operatorname{FFR}(1,4)$ is obviously better. The mean value of the optimal $B_{1}$ was $0.489 B$ and $0.530 B$ for FFR $(1,3)$ and FFR $(1,4)$ respectively, with a standard deviation of only $0.022 B$ in both cases. Thus, the choice of bandwidth allocation for both bands appears robust to the particular choice of frequency reuse scheme. While we optimize the geometric mean to ensure proportional fairness (PF), it is more telling to compare the performance of the system optimized for $\operatorname{FFR}(1,3)$ and $\operatorname{FFR}(1,4)$ in terms of throughput (defined as the sum of the rates obtained by all users) and of the coverage defined as the 5th percentile rate. Figs. 5 and 6 show the gain of $\operatorname{FFR}(1,4)$ over $\operatorname{FFR}(1,3)$ in throughput and 5 th percentile rate for the users over the 150 realizations.

From the figures, it can be seen that while the throughput is almost always impacted negatively the choice of $\operatorname{FFR}(1,4)$ over FFR(1,3), the 5 th percentile rate does noticeably better under $\operatorname{FFR}(1,4)$ compared to $\operatorname{FFR}(1,3)$ in most cases.

In a second part, we now consider 300 network realizations where the perturbation parameter $\mu=0.9$. Again, independent Rayleigh fading is assumed between all node pairs. Interestingly, $\operatorname{FFR}(1,3)$ result in a greater geometric mean rate for the

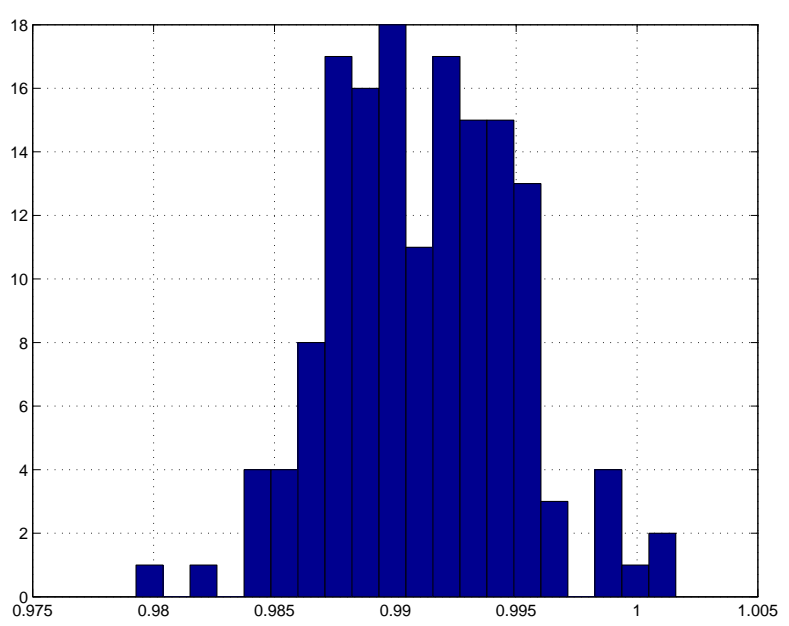

Fig. 5. Histogram of gain in throughput of users for $\operatorname{FFR}(1,4)$ over $\operatorname{FFR}(1,3)$ for no pertubation $(\mu=0)$ and 150 different fading realizations.

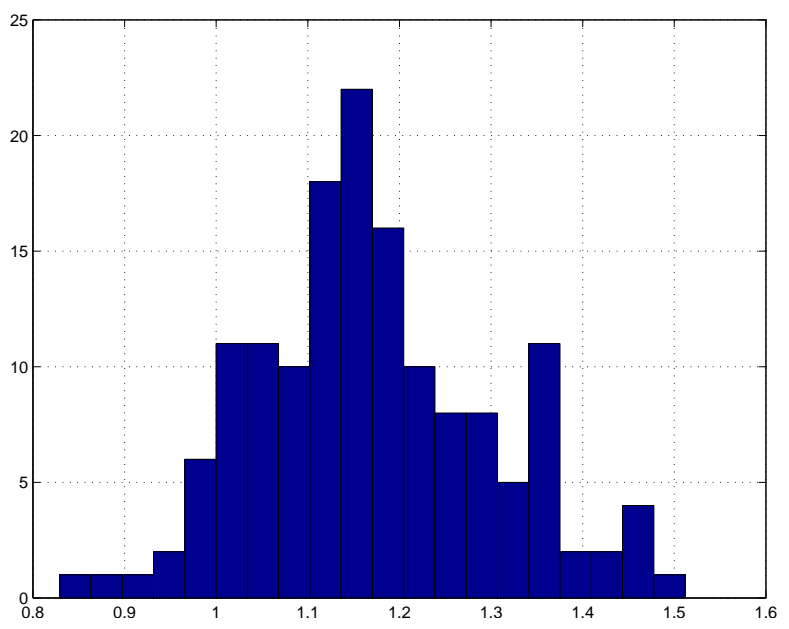

Fig. 6. Histogram of gain in 5th percentile rate of users for $\operatorname{FFR}(1,4)$ over $\operatorname{FFR}(1,3)$ for no perturbation $(\mu=0)$ and 150 different fading realizations.

users in only $26 \%$ of cases, clearly indicating that $\operatorname{FFR}(1,4)$ is preferable in the majority of cases. Fig. 7 shows a histogram of the gain in geometric mean of the rates for $\operatorname{FFR}(1,4)$ over $\operatorname{FFR}(1,3)$, confirming that $\operatorname{FFR}(1,4)$ outperforms $\operatorname{FFR}(1,3)$ in the majority of cases.

Figs. 8 and 9 show the gain of $\operatorname{FFR}(1,4)$ over $\operatorname{FFR}(1,3)$ in throughput and 5th percentile rate for the users over the 300 realizations. Clearly, the 5 th percentile rate is significantly better for $\operatorname{FFR}(1,4)$ in the majority of cases, while neither $\operatorname{FFR}(1,4)$ nor $\operatorname{FFR}(1,3)$ is obviously better in terms of throughput. The mean value of the optimal $B_{1}$ was $0.607 B$ and $0.582 B$ for $\operatorname{FFR}(1,3)$ and $\operatorname{FFR}(1,4)$ respectively, with a standard deviation of $0.06 B$ and $0.05 B$ respectively. Again, the choice of bandwidth allocation for both bands appears robust to the particular choice of frequency reuse scheme.

A natural question is how much of the preference for FFR $(1,4)$ over FFR $(1,3)$ in the perturbed base station cases was due to the perturbed geometry compared to fading. To try to answer this question, we first consider the 300 perturbed geometries that we have studied earlier (with fading). In the absence of fading, only $32 \%$ resulted in a preference for 


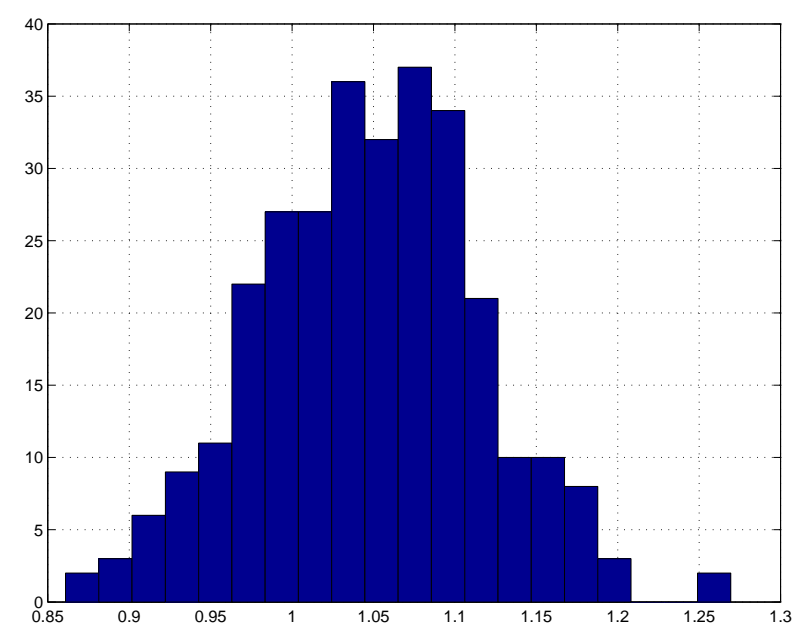

Fig. 7. Histogram of gain in geometric mean of user rates for $\operatorname{FFR}(1,4)$ over FFR(1,3) with fading and pertubation for $\mu=0.9$ for 300 different realizations.

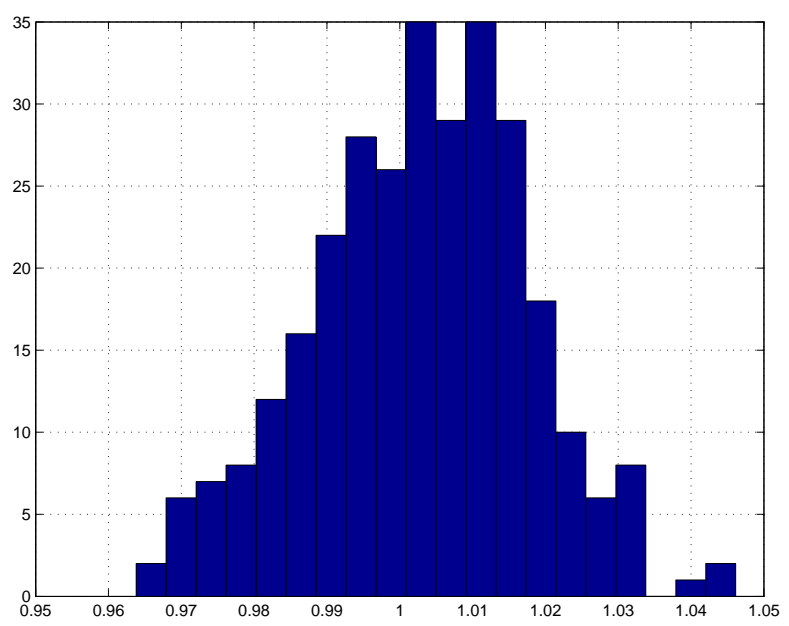

Fig. 8. Histogram of gain in throughput of users for $\operatorname{FFR}(1,4)$ over $\operatorname{FFR}(1,3)$ with fading and perturbation for $\mu=0.9$ for 300 different realizations.

FFR $(1,3)$ over FFR $(1,4)$ in terms of geometric mean of user rates. Hence clearly, the fact that the topology is not ideal is a key factor in making $\operatorname{FFR}(1,4)$ preferable to $\operatorname{FFR}(1,3)$. We also consider one specific topology. The one among the 300 realizations that resulted in the largest gain in geometric mean of user rates for $\operatorname{FFR}(1,4)$. For this specific topology, 150 new independent fading realizations were generated. It was found that in each of the 150 cases, $\operatorname{FFR}(1,4)$ outperformed $\operatorname{FFR}(1,3)$.

\section{CONClusion}

In this paper we have considered the effect of non-ideal base station locations with fading on the performance of fractional frequency reuse. Specifically, we have considered base station locations to be perturbed from their ideal hexagonal lattice positions. Results have shown that for proportional fair scheduling, $\operatorname{FFR}(1,4)$ outperforms $\operatorname{FFR}(1,3)$ which is usually assumed to be optimal. Thus, we conclude that the specific topology of the network should be considered for optimal configuration of fractional frequency reuse.

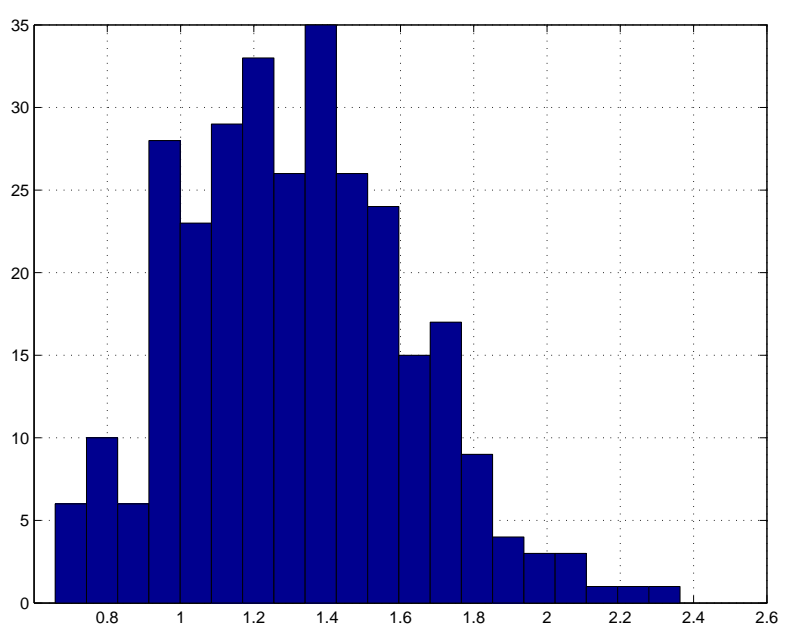

Fig. 9. Histogram of gain in 5th percentile rate of users for $\operatorname{FFR}(1,4)$ over FFR(1,3) with fading and pertubation for $\mu=0.9$ for 300 different realizations.

\section{REFERENCES}

[1] "802.16e-2005, part 16: air interface for fixed and mobiled broadband systems," Feb. 2006.

[2] 3GPP TR 25.814, "Physical layer aspects for evolved UTRA rel-7,", 2006.

[3] Alcatel, "3GPP R1-060689: System simulation results for downlink interference coordination, update," 2006.

[4] J. G. Andrews, F. Baccelli, and R. K. Ganti, "A tractable approach to coverage and rate in cellular networks," Submitted to IEEE Transactions on Communications, 2011, arXiv:1009.0516v2 [cs.IT].

[5] M. Bohge, J. Gross, and A. Wolisz, "Optimal power masking in soft frequency reuse based OFDMA networks," in "Proc. European Wireless Conf.", Aaslborg, Denmark, 2009, pp. 162-166.

[6] S.-E. Elayoubi, O. B. Haddada, and B. Fouresti/'e, "Performance evaluation of frequency planning schemes in OFDMA-based networks," vol. 7, no. 5, pp. 1623-1633, May 2008.

[7] H. S. Fhillon, R. K. Ganti, F. Baccelli, and J. G. Andrews, "Modeling and analysis of k-tier downlink heterogeneous cellular networks," Submitted to IEEE Transactions on Communications, 2011, arXiv:1103.2177v2 [cs.IT].

[8] H. Fujii and H. Yoshino, "Theoretical capacity and outage rate of ofdma cellular system with fractional frequency reuse," in Proc. IEEE Veh. Technol. Conf. - Spring, Singapore, May 2008, pp. 1676-1680.

[9] Huawei, "3GPP, R1-050507: Soft frequency reuse scheme for utran lte," 2005.

[10] X. Mao, A. Maaref, and K. H. Teo, "Adaptive soft frequency reuse for inter-cell interference coordination in sc-fdma based," in Proc. IEEE Global Telecommun. Conf., New Orleans, LA, Dec. 2008.

[11] A. Molish, Wireless Communications. Wiley-IEEE Press, 2005.

[12] L. Sarperi, M. Hunukumbure, and S. Vadgama, "Simulation study of fractional frequency reuse in WiMAX networks," FUJITSU Sci. Tech. J., vol. 44, no. 3, pp. 318-324, July 2008.

[13] A. L. Stolyar and H. Viswanathan, "Self-organizing dynamic fractional frequency reuse in OFDMA systems," Pheonix, AZ, Apr. 2008, pp. 691699.

[14] S. Toumpis and A. J. Goldsmith, "Capacity regions for wireless ad hoc networks," IEEE Trans. Wireless Commun., vol. 2, no. 4, pp. 736 - 748, July 2003.

[15] Z. Xie and B. Walke, "Enhanced fractional frequency reuse to increase capacity of ofdma systems," in 3rd International on New Technologies, Mobility and Security (NTMS 2009), Cairo, Egypt, Dec. 2009, pp. 25922595.

[16] Y. Zhou and N. Zein, "Simulation study of fractional frequency reuse for mobile wimax," in Proc. IEEE Veh. Technol. Conf. - Spring, Singapore, May 2008, pp. 2592-2595. 